Revue

Revue de l'histoire des religions

de l'histoire des religions

$3 \mid 2005$

Varia

\title{
Samson le nazir : un mythe du jeune guerrier
}

Samson the nazir: a myth of the young warrior

\section{Christophe Lemardelé}

\section{OpenEdition \\ Journals}

Édition électronique

URL : http://journals.openedition.org/rhr/4186

DOI : $10.4000 /$ rhr.4186

ISSN : 2105-2573

Éditeur

Armand Colin

Édition imprimée

Date de publication : 1 juillet 2005

Pagination : 259-286

ISBN : 2200-92086-5

ISSN : 0035-1423

\section{Référence électronique}

Christophe Lemardelé, « Samson le nazir : un mythe du jeune guerrier », Revue de l'histoire des religions [En ligne], 3 | 2005, mis en ligne le 18 janvier 2010, consulté le 10 décembre 2020. URL : http:// journals.openedition.org/rhr/4186; DOl : https://doi.org/10.4000/rhr.4186 


\section{CHRISTOPHE LEMARDELÉ}

Centre d'Études des Religions du Livre

(UMR 8584, CNRS-EPHE-Paris IV)

\section{Samson le nazir : un mythe du jeune guerrier}

L'histoire de Samson reste énigmatique tant pour son origine que pour sa signification. Il apparaît que le récit doit être qualifié de récit hérö̈que ou de mythe. En effet, Samson est typique de la figure du héros, qu'il s'agisse d'Héraklès comme de Gilgamesh : c'est à l'origine un demi-dieu accumulant les fautes. Il est contestable d'affirmer que le récit est grec car on retrouve dans le texte des motifs propres aux guerriers du livre des Juges et des livres de Samuel. Parmi les titres du héros - nazir, "sauveur» et "juge»-, seul le premier donne du sens au récit, les deux autres étant tardifs et artificiels. Mais le titre de nazir ne correspond pas au vou sacerdotal de Nombres 6 , il reflète un rite plus ancien et probablement relatif aux jeunes guerriers.

\section{Samson the nazir : a myth of the young warrior}

The origin and the meaning of the Samson's story are uncertain. In fact, it's an heroic tale or a myth because Samson is a typical hero, like Herakles or Gilgamesh: he was originally a semi-god and he accumulated faults. We cannot say that the text is Greek, since we can find in the text the motifs of the "Israelite" warrior like in the book of Judges and in the books of Samuel. Among the hero's titles - nazir, savior and judge-only the first one gives meaning to the narration of events. The other two are belated and artificial. But the title of nazir does not fit with the priestly vow of Numbers 6: it rather reflects an ancient rite which is probably linked to young warriors. 
L'histoire de Samson fait problème. Quelle est son origine : « grecque », comme certains le supposent, ou « sémitique »? Et quelle est sa signification, pour elle-même et au sein du corpus qu'est la Bible hébraïque ? En effet, comment caractériser le personnage ? Est-ce un héros, un sauveur, un juge ? Et comment expliquer la place du récit dans le livre des Juges? Est-elle plus ou moins conjoncturelle ou a-t-elle obéi à un motif précis ? La difficulté se voit d'ailleurs dans les termes utilisés pour caractériser le texte. Les exégètes allemands le qualifient de « conte » (Märchen), les anglophones usent souvent du mot «cycle» et les Français l'appellent «légende ». Mais le terme qui revient le plus régulièrement est « saga ». Nous sommes pourtant bien loin des récits islandais même si, il est vrai, la geste d'un héros aux longs cheveux y fait spontanément penser. Il est inutile pour le moment d'argumenter en faveur de telle ou telle dénomination : nous pourrons faire une proposition à la fin de l'analyse du récit. Postulant que les incertitudes relatives au récit sont sans doute dues au fait qu'il se trouve dans la Bible en effet, si ce texte appartenait à un autre corpus, on ne l'interrogerait pas de la même manière -, la présente analyse privilégiera le texte comme document autonome et non comme écrit biblique.

\section{MÉTHODE HISTORICO-CRITIQUE, ANALYSE NARRATIVE ET APPROCHES HISTORIQUES}

Le texte de Jg 13-16, relatant l'histoire de Samson de sa conception (13) à sa mort (16), en passant par son mariage (14) et divers exploits (15), a tout d'abord été passé au crible de la critique littéraire. Ainsi, des versets deutéronomistes - dus à une activité rédactionnelle et éditoriale commençant probablement dès la seconde moitié du $\mathrm{VII}^{\mathrm{e}}$ siècle à la cour de Juda et visant à la constitution de livres formant une histoire d'Israël de l'exode d'Égypte à l'exil à Babylone ${ }^{1}$ - ont

1. Pour la difficulté de faire l'histoire des royaumes d'Israël et de Juda à partir de l'historiographie deutéronomiste, voir dernièrement Nadav Na'aman, «La Bible à la croisée des sources », Annales, Histoire, Sciences sociales, $n^{\circ}$ 6, 2003, p. 1321-1346. 
rapidement été identifiés ${ }^{2}$. Tout d'abord, Jg 13,1 qui replace l'histoire de Samson, personnage issu de la tribu de Dan, dans l'histoire générale d'Israël en rappelant le mal fait par les Israélites aux yeux de Yhwh. On retrouve ce même type d'introduction tout au long du livre des Juges, avant presque chaque nouveau récit (Jg 3, 7 ; $3,12 ; 4,1 ; 6,1 ; 10,6)$. Ensuite, Jg 15, 20 et Jg 16, 31b qui concluent tous les deux que Samson fut juge pendant vingt ans. Ce titre est la marque même du rédacteur final du livre des Juges, le rôle historicothéologique donné à ce titre étant clairement énoncé en Jg 2, 18-19³. Enfin, on peut ajouter au compte du Deutéronomiste 13, 5b et 14, 4b qui mentionnent Israël : le premier de ces ajouts institue Samson comme « sauveur d'Israël » et le second précise la situation « historique » : « en ce temps-là les Philistins dominaient Israël ».

Pour le corps du récit, en revanche, il n'y a pas de consensus qui se dégage. Beaucoup d'éléments, et notamment l'insistance sur le nazir, semblent indiquer une rédaction plus tardive pour le chapitre 13 que pour le reste du récit ${ }^{4}$. Mais l'hypothèse inverse a aussi été proposée : Jg 13 ferait partie du récit originel mais pas le chapitre 16 qui relate, notamment, la capture de Samson à la suite de la tromperie de Dalila ${ }^{5}$. Une autre possibilité encore est de considérer les chapitres 13 et 16 comme compléments à un noyau ancien (14-15) : ce noyau aurait été introduit dans le livre des Juges avec l'insertion à ce moment des deux chapitres ${ }^{6}$. L'unanimité est donc

2. Voir déjà, Julius Wellhausen, Prolegomena zur Geschichte Israels, Berlin, 1886, p. 236 ss. et Martin Noth, The Deuteronomistic History, Sheffield, 1981 (Halle, 1943), p. 21 ss.

3. Ce titre permet à l'auteur judahite de préparer au mieux l'avènement ultérieur du roi David tout en critiquant le royaume du Nord par l'intermédiaire de ces juges qui échouent les uns après les autres. Pour l'analyse de cette catégorie de « juge », voir Isabelle de Castelbajac, « Les Juges d'Israël : une invention du Deutéronomiste ?», Revue de l'histoire des religions, $\mathrm{n}^{\circ} 221$, 2004, p. 83-97.

4. Voir Wolfgang Richter, Traditionsgeschichtliche Untersuchungen zum Richterbuch, Bonn, 1963, p. 140 ss. Cette position était déjà celle de Karl Budde, Das Buch der Richter, Tübingen, 1897 et de C. F. Burney, The Book of Judges, London, 1918.

5. Robert G. Boling, Judges, New York, 1975, p. 223-224 et 252-253.

6. Voir Hartmut Gese, « Die ältere Simsonüberlieferung (Richter c. 14-15) », Zeitschrift für Theologie und Kirche, $\mathrm{n}^{\circ}$ 82, 1985, p. 261-280. 
loin de se faire sur l'ensemble du texte, même si beaucoup d'exégètes optent pour une rédaction tardive du premier chapitre. Il semble cependant assuré que l'insertion de Jg 13-16 dans le livre des Juges fut tardive. Une étude menée sur le lexique a conclu à une hétérogénéité importante entre le corps du livre (Jg 3, 7 à Jg 12) et le récit de Samson - elle a conclu aussi à une homogénéité stylistique assez grande entre les différents chapitres du récit ${ }^{7}$. Mais cette insertion tardive ne signifie pas que le texte a été écrit à ce moment, les archaïsmes qu'il contient font irrémédiablement penser à une origine très ancienne impossible à préciser. Sans doute existait-il déjà et a-t-il été recopié, modifié et remis «au goût du jour ». L'insertion dans les Juges aurait dû nécessiter un travail d'édition, d'adaptation, pour rendre le récit plus conforme à l'esprit du livre, mais les retouches deutéronomistes ne sont ni nombreuses ni développées: Samson n'a pas été «historicisé ». Conservant l'apparence d'une geste héroïque, Jg 13-16 peut donc être abordé dans son ensemble.

Considérant les textes dans leur intégralité, l'analyse narrative s'est, ces dernières décennies, quelque peu opposée à la méthode historico-critique. Un des initiateurs de cette analyse narrative pour Jg 13-16, Joseph Blenkinsopp, s'est attaché à mettre en évidence les caractéristiques stylistiques du texte : le rythme poétique, les répétitions comme moyens mnémotechniques, marques possibles d'une origine orale. En outre, il a montré que le but premier du texte était littéraire puisqu'il est construit autour d'une intrigue (plot): Samson respectera-t-il son $\mathrm{vœu}^{8}$ ? Bien qu'il ne soit pas question d'un vœu dans ce récit, comme Blenkinsopp l'a présupposé, il est vrai que le lecteur garde à l'esprit le «tabou » sur les cheveux (nazir) et se doute que c'est là que réside la force de Samson. Aussi, quand ce dernier révèle le secret à Dalila, ce n'est pas une surprise pour le lecteur. Une autre étude narrative a souligné les jeux littéraires du texte : la mort du héros est annoncée dès le chapitre 13 (v. 7 :

7. Yehuda T. Radday et alii, « The Book of Judges Examined by Statistical Linguistics », Biblica 58 (1977), p. 469-499.

8. Joseph Blenkinsopp, «Structure and Style in Judges 13-16», Journal of Biblical Literature 82 (1963), p. 65-76. 
nazir jusqu'au jour de sa mort) et l'image du feu est présente et répétée, de même que la rémanence du chiffre trois ${ }^{9}$.

Si ces deux études montrent que le texte a une réelle unité stylistique et thématique (anticipations, répétitions voulues, etc.), la dernière, en revanche, en conclut exagérément et trop rapidement à l'œuvre d'art tardive sans lien direct avec une figure héroïque ancienne. Le symbolisme du feu peut avoir une origine religieuse, le nom du héros étant à rapprocher du dieu solaire Shamash. De même, les qualités littéraires ont pu croître en fonction des réécritures du texte sans penser que la première version en était dénuée. Ainsi, l'apport essentiel de l'analyse narrative est de montrer que le récit final est cohérent, les éléments importés s'étant probablement fondus dedans avec le temps; excepté les plus récents comme les mentions de « juge » et d'« Israël ».

Plus historique, l'approche de Paul Humbert a consisté à voir quatre étapes dans la formation du personnage ${ }^{10}$. Il y aurait eu à l'origine un héros profane auteur d'exploits grâce à la force détenue dans ses cheveux ${ }^{11}$. On aurait expliqué plus tard ses exploits par la présence de l'esprit de Yhwh en lui. Ensuite, on aurait fait le lien entre le héros chevelu et les nezirîm, consacrés à Yhwh et devant laisser leurs cheveux longs. Enfin, on aurait fait de Samson un juge délivrant Israël des Philistins : le héros local serait devenu héros national. La reconstruction peut sembler trop limpide mais elle permet de donner une explication plausible à une figure si peu biblique.

La dernière approche que nous évoquerons fut celle d'Othniel Margalith qui, lui, chercha à trouver l'origine de l'histoire de Samson sans en reconstruire l'évolution. Sa démarche a consisté à relever et à analyser les parallèles grecs de l'histoire du héros biblique.

9. Robert Alter, «Samson without Folklore », dans S. Niditch (ed.), Text and Tradition. The Hebrew Bible and Folklore, Atlanta, 1990, p. 47-56.

10. Paul Humbert, «Les métamorphoses de Samson ou l'empreinte israélite sur la légende de Samson », Revue de l'histoire des religions, ${ }^{\circ}$ 80, 1919, p. $154-170$.

11. Gunkel partageait les mêmes présupposés d'une origine profane du héros : il aurait été le représentant d'une nature sauvage en conflit avec la sphère culturelle qu'était la civilisation philistine. Hermann Gunkel, «Simson », dans Reden und Aufsätze, Göttingen, 1913, p. 38-64. 
Par exemple, l'épisode des abeilles et du miel dans la carcasse du lion tué par Samson n'a pas d'équivalent dans la mythologie orientale, alors que la croyance selon laquelle les essaims d'abeilles naissaient dans les cadavres d'animaux est clairement attestée dans le monde $\operatorname{grec}^{12}$. De même pour les boucles magiques de Samson: on ne trouverait de parallèles que dans la culture grecque avec, notamment, l'histoire du «cheveu d'or» rendant immortel le roi Pterelaus ${ }^{13}$. Mais ce serait principalement la figure d'Héraklès qui apparaîtrait comme un sosie en plus grand de Samson, au point d'en être le modèle ${ }^{14}$. Enfin, il n'est pas certain que Dan, la tribu de Samson, ait été sémitique. La mention de «Denye(n) » parmi les Peuples de la Mer, sur l'inscription de Medinet Habu commémorant la victoire de Ramsès III (première moitié du XII ${ }^{\mathrm{e}}$ siècle), invite à penser qu'il s'agirait des Danites et qu'ils pouvaient être ainsi très proches culturellement des Philistins ${ }^{15}$. Margalith n'hésite pas à faire ces rapprochements et à relier ainsi, par l'intermédiaire des Denye(n), les Danaoï grecs ${ }^{16}$ aux Danites sémitiques ${ }^{17}$. Mais, comme nous allons le voir, cette possible origine « grecque » n'est pas sans poser de problèmes. En outre, elle ne nous fait pas mieux saisir le sens du récit.

Pour résumer, nous avons un texte d'une assez grande cohérence narrative comportant des ajouts deutéronomistes. L'histoire rédactionnelle du corps du récit de même que son origine et l'évolution

12. Voir la figure mythique d'Aristée, dont l'histoire est racontée comme un mythe étiologique par Virgile, Géorgiques IV, 317 ss.

13. Apollodore, Bibliothèque II. 4, 7 ss. Voir Othniel Margalith, «Samson's Riddle and Samson's Magic Locks », Vetus Testamentum, ${ }^{\circ} 36$, 1986, p. 225-234.

14. O. Margalith, "More Samson Legends", Vetus Testamentum 36 (1986), p. 397-405 ; «The Legends of Samson/Heracles », Vetus Testamentum, n 37,1987 , p. 63-70.

15. Pour cette question, voir Allen H. Jones, Bronze Age Civilisation. The Philistines and the Danites, Washington, 1975.

16. Nom qui est l'équivalent des Achéens dès le début de l'épopée homérique : Homère, Iliade I, 42 ss.

17. O. Margalith, The Sea Peoples in the Bible, Wiesbaden, 1994 (Tel-Aviv, 1988), p. 115-124. 
du personnage de Samson sont difficiles à cerner ${ }^{18}$. Mais il importe également de connaître la signification culturelle de ce texte. Commençons donc notre analyse.

\section{SAMSON HÉROS}

Avant toute chose, il nous faut signaler la probable ascendance divine de Samson, à l'instar d'autres héros mythiques. Sans aller jusqu'à considérer qu'en Jg 13 l'épisode du messager de Yhwh, qui vient voir la femme dans le champ en l'absence de son mari, peut être l'écho d'un autre récit dans lequel une divinité fécondait cette femme ${ }^{19}$, il reste que la conception de Samson est tout à fait miraculeuse. En effet, contrairement au récit de conception de Samuel, qui est assez comparable, il n'est pas dit que le «père », Manoah, connut sa femme. Même relu ou conçu par un auteur yahwiste, il reste la trace d'une origine divine en Jg 13. Or celle-ci n'est pas difficile à deviner avant l'interprétation yahwiste: Shamash, puisque Shimshôn peut signifier « petit Shamash » - et non exactement « petit soleil », il ne représenterait pas l'astre solaire mais plus vraisemblablement la divinité. La présence de la ville de Beth Shèmèsh à proximité du territoire de Dan, avant sa migration vers le nord (Jg 17-18), prouve d'ailleurs que le culte de Shamash/ Shèmèsh était présent dans la région ${ }^{20}$. À l'instar d'autres héros d'ascendance divine, ses exploits, particulièrement les derniers,

18. Pour une critique de tous ces travaux - excepté les approches historiques de Humbert et de Margalith -, voir Jichan Kim, The Structure of the Samson Cycle, Kampen, 1993, p. 1-114. Cet auteur se situe du côté de l'analyse narrative et veut montrer que le texte est à l'origine une poésie narrative très structurée - il est possible en effet que ce récit ait existé sous cette forme avant son introduction dans le livre des Juges.

19. Pour cette interprétation en se fondant sur des récits grecs, voir O. Margalith, « More Samson Legends », p. 397-402.

20. Ce qui va à l'encontre de l'hypothèse « mycénienne » de Margalith. À noter la suggestion très intéressante que fit Burney: Shamash étant au Proche-Orient le dieu de la justice - le soleil qui est au-dessus de tout et voit donc tout - et Dan signifiant « juge », le nom de la tribu pourrait ainsi se référer à cette divinité. $C f$. C. F. Burney, Judges, p. 392. 
sont la marque des géants : prendre les portes de Gaza et les transporter bien plus à l'est, sur la montagne en face d'Hébron (Jg 16, 1-3), ne peut être effectué par un homme, même d'exception ${ }^{21}$. Peutêtre, dans le récit originel de Jg 13-16, Samson était-il décrit comme Gilgamesh : un homme aux allures gigantesques (T. I, § 4, I, 50-II, 1-8 ${ }^{22}$. De même, en ce qui concerne l'épisode de la source en Jg 15, 18-19, dans lequel Elohim fend le bassin pour donner à boire à Samson, il est fort probable que, dans une version antérieure, il n'ait pas eu besoin d'une aide divine pour se procurer à boire - Gilgamesh, dans sa fureur après la mort d'Enkidu, « creuse des puits» (T. X, § 1, I, 3). Enfin, l'épisode final a également un aspect gigantesque, quand Samson saisit les colonnes de l'édifice des Philistins et le fait s'écrouler. Mais pour l'établir comme héros, il y a d'autres aspects à souligner.

L'histoire proprement dite ne débute qu'à la fin du chapitre 13, quand l'enfant a grandi et que l'esprit - le «souffle»- de Yhwh commence « à l'agiter » dans le camp de Dan (v. 25). Samson n'est donc plus alors un enfant, sans être encore un homme. C'est un jeune homme qui, dès le début du chapitre 14 , se rend à Timna, la ville philistine la plus proche du territoire de Dan : il y voit une femme et veut immédiatement l'épouser. Suivent alors les différents exploits et péripéties du héros : le combat avec le lion, l'énigme posée aux Philistins de Timna, les trois cents renards qui brûlent les cultures philistines, la bataille à Ramat-Lehi gagnée avec une mâchoire d'âne, etc. Or, si Samson est à juste titre connu pour sa force de colosse, celle-ci apparaît aussi comme étant une force nerveuse, voire furieuse. C'est le cas notamment lorsqu'il « déchire » le lion comme un chevreau. Il est également un personnage rusé. Quand il empoigne les renards et met le feu à leur queue pour détruire toutes les cultures des Philistins, il fait montre d'un esprit

21. Et ce n'est pas «Dieu» qui permet l'exploit, Samson n'ayant besoin d'aucune aide divine pour cet exploit : il n'y a ni Elohim ni souffle de Yhwh. Le style si concis de Jg 16, 1-3 peut laisser supposer qu'il fait partie, d'un point de vue uniquement formel, des strates les plus anciennes du texte.

22. Pour l'établissement du texte et la traduction, Raymond-Jacques Tournay, Aaron Shaffer, L'Épopée de Gilgamesh, Paris, 1994. 
malin et malfaisant $(15,3)$. De même lorsque, aveugle, il demande au garçon qui le guide de le placer entre les colonnes de l'édifice philistin pour le détruire alors qu'on le croit sans force. Samson n'est donc pas qu'une brute épaisse, il n'est pas Polyphème. On peut le définir comme un personnage colérique et instinctif. Après que sa femme l'a trompé en révélant aux Timnites la solution à son énigme, Samson rentre chez son père enflammé de colère : "sa narine brûlait » $(14,19)$. Il est impulsif, répudiant sa femme aussi rapidement qu'il l'a épousée et, finalement, voulant la reprendre peu après $(15,1)$. En outre, il est violent sans avoir de scrupules : sans foi ni loi! Pour respecter sa promesse, lorsque les Timnites trouvent la solution à son énigme, il tue trente innocents d'Ashqelon afin de les dépouiller $(14,19)$. Cette violence est voisine d'une certaine sauvagerie, n'est-il pas le « maître des animaux », qu'il s'agisse de lions comme de renards? Ne réside-t-il pas un temps dans une grotte $(15,8)$ ? Il se tient d'ailleurs toujours à l'écart des lieux civilisés, ne se rendant dans une ville que pour voir une prostituée $(16,1)$. Il se tient même à l'écart des chemins. Quand il repart de Timna, après avoir vu sa future femme, il fait un détour pour voir le cadavre du lion tué $(14,8)$. Lors de sa conception, quand sa mère veut revoir le messager d'Elohim, elle se tient elle-même à l'écart, loin de son foyer : dans un «champ » $(13,9)$. Il faut noter encore que Samson ne se tient pas seulement à la frontière de la sauvagerie et de la civilisation, mais également à la frontière politique entre les Danites et les Philistins.

Samson est donc l'homme des marges et des écarts, et dans tous les sens du terme. Sa conduite est loin d'être irréprochable, sa faiblesse auprès des femmes est patente. Il se rend chez une prostituée puis, juste après, tombe amoureux de Dalila $(16,4)$. Il est doublement faible avec les femmes puisqu'à deux reprises elles réussissent à lui arracher un secret, d'abord la solution de l'énigme $(14,17)$, puis le secret de sa force $(16,17)$. Il commet des fautes en ne respectant pas les deux interdits du nazir énoncés en Jg 13 - cet état de nazir semblant être la condition, dans ce chapitre à l'allure de conte, pour que la femme stérile puisse enfin concevoir. En effet, il mange le miel rendu impur à cause du cadavre $(14,9)$ et fait un «festin », 
(mishtèh de shâtâh, « boire »), au cours duquel il boit probablement du vin $(14,10)$. Se rendant pour la première fois dans une ville, il s'est d'ailleurs mis en danger en s'approchant «des vignes de Timna » $(14,5)$. Nous pouvons ajouter à ce non-respect des règles du nazir, l'imprudence de Samson quand il saisit par inadvertance la mâchoire d'âne «fraîche » $(15,15)$, pour vaincre les Philistins. Même si contracter une impureté par le contact d'un cadavre n'est pas mentionné parmi les interdits du nazir dans ce texte, le fait que ce détail soit mentionné montre que Samson ne sait pas distinguer le pur de l'impur. Mais la faute la plus importante du héros est bien entendu de révéler le secret de sa force. Il dit à Dalila qu'il est nazir et lui explique ce que cela signifie, se mettant ainsi à la merci de ses ennemis ${ }^{23}$.

La relation entre les interdits du nazir et les fautes du héros est souvent contestée par les exégètes mais, d'un strict point de vue littéraire, il faut bien admettre que la manducation du miel trouvé dans un cadavre fait bien écho à l'interdiction préalable de manger quelque chose d'impur. Ces fautes préexistaient sans doute aux interdits puisque, excepté dans le cas du miel, la relation n'est pas directe. En effet, l'épisode de la mâchoire fraîche n'a pas entraîné d'interdit spécifique et l'épisode du banquet a été conservé, seulement augmenté d'un commentaire du narrateur (Jg 14, 10b) afin de tenter de surmonter la contradiction avec l'interdit sur le vin énoncé en Jg 13. Ainsi, les interdits de nazir se seraient appliqués à un texte relatant les fautes d'un héros, s'adaptant au récit en faisant, pour seulement un cas, d'une faute un interdit - le miel impur.

23. C'est l'épisode le plus connu du récit, la scène de la coupe des cheveux ayant inspiré les plus grands peintres (van Dyck, Rembrandt). L'histoire est d'ailleurs bien souvent célèbre comme étant celle de "Samson et Dalila » voir l'opéra de Saint-Saëns au livret très librement inspiré du texte biblique. Auparavant, il semble que le récit n'était pas reçu ainsi. On peut notamment voir au Louvre (salle Suger) des objets en ivoire datant du Moyen Âge qui représentent le héros $e t$ ses divers exploits : un peigne du $\mathrm{IX}^{\mathrm{e}}$ siècle sur lequel Samson (plutôt que David) ouvre de ses mains la gueule d'un lion pour le déchirer, un pion de tric-trac du XII ${ }^{\mathrm{e}}$ siècle avec le héros tenant quatre renards dans une main et une torche dans l'autre, enfin deux petits panneaux de coffret de la même époque, l'un représentant Samson massacrant les Philistins à l'aide de la mâchoire d'âne, l'autre narrant en trois scènes successives l'épisode avec Dalila. 
Quoi qu'il en soit, Samson est l'homme des écarts et ces écarts sont propres aux jeunes hommes. Car le héros se caractérise avant tout par sa jeunesse. Comme nous l'avons vu, l'esprit de Yhwh commence à l'agiter dès ce moment-là. Il semble également encore bien jeune quand il veut se marier, c'est pourquoi il demande à son père de prendre la femme pour lui $(14,3)$. Lorsqu'il descend à Timna, le lion qu'il tue est, comme lui, « un jeune lion» $(14,5)$. Et lorsqu'il festoie avec les Philistins de Timna - ils sont trente « compagnons »-, le rédacteur intervenant pour sauvegarder la cohérence du récit dit: «car c'est ainsi que font les jeunes gens (bachûrîm)» $(14,10 b)$. C'est d'ailleurs probablement cette jeunesse - et cet esprit de jeunesse qu'il conserve tout le long du récit - qui lui fait commettre des fautes et qui le rend si impulsif. Samson est tout sauf sage, ses actions d'éclat, loin de « sauver Israël », mettent en danger la tribu de Juda $(15,10)$. Tout ceci confirme l'idée que l'appellation « sauveur d'Israël » est due à un rédacteur tardif, de même que le titre de juge car, curieusement, les Danites n'entrent jamais en action tant Samson agit en solitaire : il n'est pas le moins du monde un chef ! Le seul qualificatif que l'on peut lui attribuer est celui de « héros » car il en a toutes les qualités et tous les défauts.

\section{LE TYPE DU HÉROS}

Les exploits dus à la force et à la ruse, l'intempérance, les fautes, la jeunesse sont les signes du héros. Contrairement à ce que pense Margalith, ils ne sont pas propres au seul Héraklès. Gilgamesh est également un tueur de lions : « et dans les défilés de la montagne, j'ai tué des lions» (T. X, §2, I, 34). Le héros mésopotamien a également des accès de fureur suite à la perte de son ami Enkidu et, à l'instar de celui-ci, devient presque sauvage à son tour (T. X, § 1, I, 1-4). Il n'est pas non plus sans commettre des erreurs à cause de son intempérance, brisant les ancres de pierre qui permettent au bateau de franchir les «eaux de la mort» (T. X, § 7, II, 35-44) ou se faisant ravir par négligence la plante de jouvence (T. XI, § 14, VI, 285-289). La jeunesse de Gilgamesh semble aussi évidente 
puisqu'au début de l'épopée, il combat les jeunes gens d'Uruk (T. I, $\S 6, \mathrm{I}, 12$ ) et prend toutes les jeunes femmes à leur mari, obligeant le peuple à en appeler aux dieux : "C'est un buffle impétueux que tu as créé dans Uruk-l'Enclos : rien de comparable au choc de ses armes. Attentifs à lui, ses compagnons restent debout; il opprime les guerriers d'Uruk comme un tyran. Gilgamesh ne laisse pas de fils à son père, jour et nuit, il se déchaîne avec violence ; c'est pourtant lui, le pasteur d'Uruk-l'Enclos, Gilgamesh, lui, leur pasteur, et eux, son peuple (?). Puissant, superbe, avisé... Gilgamesh ne laisse pas de fille (à son mari) qu'elle soit fille d'un guerrier ou compagne d'un jeune homme » (T. I, § 7, VI, 27-39). C'est pourquoi les dieux créent Enkidu, plus sauvage et plus fort que lui ${ }^{24}$, pour le vaincre et pour qu'il s'attache à lui afin qu'à sa mort, Gilgamesh apprenne ce qu'est la vie, c'est-à-dire qu'il prenne conscience de sa condition mortelle - il ne devient « avisé » qu'à la suite de toute cette aventure. L'épopée est donc initiatique comme on peut le dire aussi, dans une moindre mesure, de l'histoire de Samson qui ne devient adulte qu'en étant prisonnier et aveugle, implorant la divinité et avouant ainsi sa faiblesse et sa condition mortelle - soumis !

Ainsi, si les parallèles existant entre Samson et Héraklès sont importants, ils ne le sont pas moins entre le héros biblique et Gilgamesh. Si l'on se tient à une position diffusionniste des mythes, et s'il faut penser que Samson dépend d'Héraklès, il faut alors convenir que ce dernier pourrait dépendre de Gilgamesh ${ }^{25}$. Mais les parallèles ne se font pas qu'entre ces trois héros. On retrouve en effet toutes ces caractéristiques chez d'autres héros, qu'il s'agisse de la force comme de l'intempérance ou, pour allier les deux, de la fureur ${ }^{26}$. Héraklès en

24. C'est une prostituée qui civilise Enkidu (T. I, § 13). On peut voir là un parallèle avec Dalila qui « adoucit» Samson.

25. Surtout si l'on ajoute ce genre de détail : après la mort de son compagnon Enkidu, le héros se lamente et dit : «revêtu d'une peau de lion, je parcourrai la steppe ! » (T. VIII, §6, III, 9). Ce motif, si présent dans la tradition sur Héraklès, ne se retrouve pas dans le récit sur Samson!

26. Le héros irlandais Cuchulainn est pris de fureur lors de son premier combat. Comme Samson, il est tout jeune encore et se bat à la frontière de son pays. Au retour, son peuple est obligé de le plonger dans l'eau froide pour le calmer. Voir Georges Dumézil, Heur et malheur du guerrier. Aspects mythiques de la fonction guerrière chez les Indo-Européens, Paris, 1985 (1969), p. 23-24. 
est le modèle grec mais d'autres héros grecs et non-grecs ont des traits similaires, même moins accentués. Dans le premier chant de l'Iliade, lorsque la querelle entre Achille et le roi Agamemnon éclate, ce dernier décrit ainsi le héros : "Ton plaisir toujours, c'est la querelle, la guerre et les combats » (I, 177). Il ajoute plus loin : «Si les dieux toujours vivants ont fait de lui un guerrier, lui donnent-ils donc mission pour cela de ne s'exprimer qu'en injures? » (I, 290-291). Ce héros est un demi-dieu - Achille est fils de Thétis - et il est en quête d'immortalité mais se contente à défaut de gloire : « $\mathrm{O}$ mère, si tu m'as enfanté pour une vie trop brève, que Zeus Olympien qui tonne sur les cimes me donne au moins la gloire ! » (I, 352-354). Héraklès, fils de Zeus, obtient l'immortalité après bien des épreuves et des douleurs ${ }^{27}$, alors que Gilgamesh, pourtant au deux tiers divin (T. I, § 4, I, 46), rentre « bredouille » de sa quête du secret de la vie. Que la geste du héros se termine ou non en apothéose, il reste qu'il devient rarement une divinité céleste à part entière, l'aspect chthonien l'emportant le plus souvent ${ }^{28}$.

Surhomme ou «sous-dieu », le héros ne sait où se situer, il ne peut être un homme comme les autres, il en vient à les mépriser et à espérer devenir une divinité. Mais il n'est souvent que l'enjeu de la rivalité des dieux. C'est le cas du héros scandinave Starkadr, auquel on accorde trois vies mais au cours desquelles il commettra trois fautes: serviteur des rois, il trompe ou tue lui-même ses maîtres ${ }^{29}$. Il n'est pas utile d'insister sur les épreuves que le héros doit endurer, les quêtes qu'il doit mener. Pour Samson, si l'on exclut le rôle de sauveur d'Israël trop tardif, l'épreuve semble être le respect des interdits de nazir, véritable défi pour ce héros au caractère insoumis. Enfin, quand le héros s'apprête à mourir, c'est un jeune homme qui est chargé de la tâche, soit en le décapitant dans le cas de Starkadr, soit en allumant le bûcher dans celui d'Héraklès. Le héros a vieilli et, en quelque sorte, se retrouve face

27. Hésiode, Théogonie, 950-955.

28. Gilgamesh est « seigneur des enfers ». Voir R. J. Tournay, A. Shaffer, L'Épopée de Gilgamesh, p. 20 ss.

29. G. Dumézil, Mythe et épopée II. Types épiques indo-européens : un héros, un sorcier, un roi, Paris, 1986 (1971), p. 23-58. 
à lui-même, jeune. Dans le récit de Samson, il y a aussi un jeune homme, un «adolescent» (na'ar), à qui le héros demande de le guider entre les colonnes pour aller vers sa mort $(\mathrm{Jg} 16,26)$. Cette mention permet d'insister sur le caractère toujours jeune du héros : refusant le monde des hommes confirmés, il ne se reconnaîtrait que dans celui des jeunes qu'il n'a jamais vraiment quitté.

De nombreux travaux ont été menés sur le monde grec ancien afin de comprendre les relations entre les récits de la mythologie et la réalité rituelle, pressentant qu'il y avait un rapport entre les héros et des institutions de rites de passage liées à la guerre ${ }^{30}$. Finalement, influencé par l'anthropologie structurale de Claude Lévi-Strauss, Pierre Vidal-Naquet a montré l'existence de ce rapport. En analysant un mythe étiologique lié à la fête des éphèbes athéniens qui devaient un service militaire à la cité, il a mis en évidence le caractère «inversé » du jeune guerrier. Le blond Xanthos, champion des Béotiens, est opposé au noir Mélanthos, champion des Athéniens, sur la frontière territoriale des deux cités. Ce dernier remporte le combat non par sa bravoure mais par ruse : le héros-guerrier se montre déloyal. Le modèle du jeune guerrier n'était pas le soldat discipliné et lourdement armé, l'hoplite, mais le chasseur rusé ${ }^{31}$.

À Athènes, le modèle du jeune guerrier a été sauvegardé à l'état de mythe, le rite de passage, constitué par l'éphébie dans la cité démocratique, ayant évolué en fonction des transformations politiques de la cité. À Sparte, en revanche, dans la cité à l'évolution historique « opposée », le rite de passage a été conservé et même systématisé ${ }^{32}$. Les jeunes garçons étaient élevés ensemble dans des conditions très dures - spartiates ! - et quand ils étaient devenus de jeunes hommes, certains d'entre eux étaient choisis pour être des «cryptes».

30. Voir notamment Henri Jeanmaire, Couroi et Courètes. Essai sur l'éducation spartiate et sur les rites d'adolescence dans l'antiquité helléni$q u e$, Lille, 1939 ; Francis Vian, «La fonction guerrière dans la mythologie grecque », dans J.-P. Vernant (dir.), Problèmes de la guerre en Grèce ancienne, Paris-La Haye, 1968, p. 53-68.

31. Pierre Vidal-Naquet, «Le chasseur noir et l'origine de l'éphébie athénienne », dans Le chasseur noir. Formes de pensée et formes de société dans le monde grec, Paris, 1991 (1981), p. 151-175.

32. P. Vidal-Naquet, « Le cru, l'enfant grec et le cuit », Ibid., p. 177-207. 
Pendant un temps déterminé, ils devaient survivre en dehors de la cité et pouvaient commettre larcins et crimes, notamment assassiner des Hilotes, peuple soumis aux Lacédémoniens. Le rite de passage est là patent puisque les cryptes étaient séparés avant d'être agrégés ensuite à la société des hommes d'exception. Errant dans la nature hostile, peu armés et agissant la nuit sans aucun esprit de loyauté, ils mettaient finalement en pratique les récits héroïques. Ce type d'institution n'est pas propre à la Grèce antique et archaïque, c'est un fait anthropologique attesté dans beaucoup de sociétés à un moment de leur histoire ou selon qu'elles étaient plus ou moins guerrières ${ }^{33}$.

La question qui se pose désormais est : qu'en est-il de Samson? Nous sommes partis de la figure du héros et nous voilà à celle du guerrier et du rite qui l'a formé comme tel. Nous sommes partis des récits mythiques et nous voici aux rites de passage. Nous trouvons en effet bien des parallèles entre Samson et d'autres héros : la jeunesse, l'intempérance, le manque de loyauté, les fautes, les marges (frontière, vie sauvage), la puissance sexuelle mais la faiblesse envers les femmes. Se pourrait-il qu'il en soit de même pour les rites ou les institutions ? Avant toutefois de formuler toute hypothèse, il nous faut tenir compte d'un conseil : « [...] lorsqu'on a à comparer les mythes et légendes qui illustrent chez divers peuples la fonction guerrière, on constate souvent que des scènes homologues, par exemple une lutte contre un genre d'adversaire particulièrement fort ou effrayant, ont été employées sans grande variation ici dans un récit d' « initiation », à la gloire du héros novice, là dans un récit de «confirmation » ou de "promotion », à la gloire d'un héros déjà éprouvé. [...] L'exégète ne doit pas oublier cette donnée élémentaire et se gardera de généraliser les notions de Jünglingsou de Kriegerweihe ${ }^{34}$. Il faut donc se garder de toute interprétation

33. Pour une étude ethnologique de ce type d'institution, mettant en évidence le rapport étroit entre la figure du grand guerrier spécialiste des combats singuliers et les grandes cérémonies d'initiation masculine, voir Maurice Godelier, La production des Grands hommes. Pouvoir et domination masculine chez les Baruya de Nouvelle-Guinée, Paris, 1982.

34. G. Dumézil, Heur et malheur, p. 178. 
précipitée ou forcée ; de même qu'il nous faut revenir à l'histoire de Samson et aux textes bibliques afin d'éviter toute comparaison abusive.

\section{SAMSON GUERRIER}

Les textes bibliques ne sont pas riches en récits pouvant évoquer des institutions guerrières. Cependant, la figure du guerrier est bien présente et Samson a des qualités propres aux figures de guerriers présents dans la Bible hébraïque même, relativisant son aspect atypique et sa possible origine étrangère. En effet, les exploits de Samson ne sont pas tous du même type. Son premier exploit est propre au héros, nous l'avons vu pour Gilgamesh et pour Héraklès : il tue un lion. Mais cet exploit est propre aussi au guerrier et, qui plus est, au guerrier israélite. En effet, Benayahou, cité parmi les guerriers de David, abat un lion dans une citerne un jour de neige (2 S 23, 20). Sans doute était-il le seul à être assez téméraire pour aller combattre l'animal dans un lieu sombre et clos. De même, il est attribué à David dans sa jeunesse d'avoir protégé les troupeaux de moutons contre les lions et les ours (1 S 17, 34-36). Ainsi, la mention de Samson tuant un lion n'est pas si extraordinaire et n'est ni particulièrement grecque ni particulièrement mésopotamienne.

Un autre épisode dans l'histoire de Samson est un motif de guerrier bien présent dans la Bible : il s'agit de la bataille de Lehi. Les Judahites s'apprêtent à livrer Samson aux Philistins quand celui-ci «brûle » ses liens, saisit une mâchoire d'âne et abat mille hommes (15, 14-16). Or, le motif du guerrier seul et à découvert au milieu de l'armée ennemie se retrouve dans la mention si ténue de Shamgar: «il battit les Philistins au nombre de six cents avec un aiguillon à bœufs » $(\mathrm{Jg} 3,31)$. Shamgar est aussi nommé dans le chant de Debora $(\operatorname{Jg} 5,6)$ et porte la même ascendance divine : fils de 'Anath. Si l'on interprète le peu de données d'un point de vue uniquement historique, on peut alors voir ce personnage comme un mercenaire 'Apiru au service de Ramsès III. Il aurait combattu les Philistins en Palestine du nord pour le compte du pharaon et 
porterait le nom de la divinité ougaritique parce que son culte se serait développé de Syrie jusqu'en Égypte ${ }^{35}$. Mais il est possible également que le guerrier ait été un héros du même type que Samson, un demi-dieu fils de cette déesse guerrière - son exploit s'expliquant précisément par cette ascendance -, dont les récits n'auraient pas été conservés et repris. Il est possible aussi qu'il y ait eu contamination de l'un à l'autre héros. En effet, Shamgar, comme Samson, tue des Philistins, et ceci de la même manière. Peut-être l'épisode de Lehi a-t-il été à l'origine l'exploit du seul Shamgar. Il y a d'ailleurs une autre occurrence troublante à propos de Philistins tués de la même manière mais cette fois par un dénommé Shamma. Ce dernier reste au milieu d'un champ cultivé pour le protéger contre les Philistins alors que l'ensemble de son armée s'est enfui (2 S 23,11-12). Contrairement à Samson, Shamma n'est pas destructeur de cultures ${ }^{36}$, mais il incarne toutefois la figure du guerrier surhumain capable de vaincre à lui tout seul une armée. Ainsi, les deux héros ont pu nourrir le récit de Samson - à moins que ce ne soit ce dernier qui ait inspiré les deux autres mentions -, car Shamma se bat lui aussi à Lehi. Il faut ajouter aussi les mentions de Ishbaal tuant huit cents hommes et de Abishaï en tuant trois cents avec leur seule lance (2 S 23, 8, 18). Le motif de l'homme seul contre une armée est donc bien celui du grand guerrier et il est très présent dans les textes bibliques les plus archaïques.

Si l'histoire de Samson contient ce motif, elle n'a pas en revanche un autre motif bien connu qui est celui du duel en apparence démesuré. Le plus connu est évidemment le combat opposant David à Goliath, le jeune homme à la fronde contre le géant surarmé

35. Nili Shupak, «New Light on Shamgar ben 'Anath », Biblica, $n^{\circ} 70$, 1989 , p. $517-525$.

36. Il y a d'ailleurs un parallèle grec : lors des guerres médiques, et précisément lors de la bataille de Marathon, Echetlaios, un héros inconnu tout d'abord, fait des ravages dans les rangs des Perses avec son manche de charrue (Pausanias, Description de la Grèce, I, 32, 5). Mais les notices hébraïques de ces héros guerriers ( $2 \mathrm{~S} 21,15-22$ et $23,8-39$ ) sont bien plus anciennes, leur style et les variantes textuelles attestant la difficulté des copistes pour les comprendre. Voir André Caquot, Philippe de Robert, Les Livres de Samuel, Genève, 1994, p. 587-589 et 619-625. 
(1 S 17,41ss). Il est probable d'ailleurs que l'on ait attribué à David l'exploit d'un nommé Elhanan $(2 \mathrm{~S} 21,19)$ puisque tout le monde s'accorde sur le fait que le géant philistin a été nommé Goliath à partir de cette brève mention. Il reste que le motif du héros battant un homme d'aspect gigantesque avec une arme rudimentaire était répandu ${ }^{37}$, puisque c'est ainsi que Benayahou tue un Égyptien : à l'aide d'un bâton (2 S 23, 21). Comme David - ou plutôt David comme Benayahou - il affronte le géant après avoir battu les deux « héros » de Moab et après avoir tué le lion.

Si nous n'avons pas dans la Bible d'autres récits de héros comme celui de Samson, en revanche, les figures de grands guerriers, connus pour quelques faits d'armes, abondent et leurs exploits sont tout aussi impressionnants. Et Samson, par son combat victorieux contre le lion et par sa démonstration de force contre les Philistins, apparaît donc également comme un modèle de grand guerrier, même s'il n'y a pas de combat singulier parmi ses exploits.

Mais Samson n'est pas seulement conforme à la figure du guerrier accompli, il est aussi un jeune guerrier. Le fait de tuer un jeune lion, et que ce soit son premier exploit, peut nous incliner à penser que l'animal permet au jeune guerrier de montrer sa bravoure. La notion de rite de passage n'est donc pas à négliger ${ }^{38}$, malgré le peu d'informations dont nous disposons, sans bien sûr vouloir l'appliquer à tout prix à la « société israélite » : le modèle anthropologique existe mais il doit être utilisé avec des précautions et des nuances ${ }^{39}$.

37. C'est un motif tout autant grec que sémite, tout aussi fréquent dans l'Iliade que dans nos récits hébraïques et que l'on retrouve également dans les mythes ou dans des références mythiques, qu'il s'agisse des dieux Marduk, Ba'al et même Yhwh dans le rôle du champion. Voir Roland de Vaux, «Les combats singuliers dans l'Ancien Testament », dans Bible et Orient, Paris, 1967, p. 217-230.

38. Il faut cependant distinguer les grands rites institués des rites mineurs dans lesquels on retrouve la fameuse structure ternaire : séparation-margeagrégation. Pour une redéfinition des rites de passage, voir Pierre Bourdieu, «Les rites d'institution», dans Ce que parler veut dire, Paris, 1982, p. 121-134; bien que l'auteur ne fasse pas de distinction entre de vrais rites et de simples actes d'institution.

39. Pour des réserves, voir dernièrement Claude Calame, « Le rite d'initiation tribale comme catégorie anthropologique (Van Gennep et Platon) », Revue de l'histoire des religions, $\mathrm{n}^{\circ} 220,2003$, p. 5-62. 


\section{SAMSON NAZIR}

Contrairement aux types de rites connus encore en Grèce à l'époque classique, nous ne disposons pas d'un rituel clairement attesté comme à Sparte ou d'un mythe étiologique rappelé au moment d'une fête comme celle des éphèbes athéniens. À première vue, nous n'avons que le récit d'un héros reconnaissable à sa jeunesse, sa brutalité sauvage, sa force, son manque de loyauté, etc. Il y a toutefois un élément souvent négligé et qu'il faut étudier de près : Samson est dit nâzîr.

Pour beaucoup d'exégètes, l'attestation de nazir en Jg 13, 5.7 et 16, 17 fait partie des derniers ajouts au texte. On remarque en effet une grande parenté entre les interdits énoncés dans le premier chapitre de l'histoire de Samson et les interdits pour ce rituel contenus dans le texte sacerdotal de $\mathrm{Nb} 6$ : dans l'un comme dans l'autre, il y a l'interdit du vin, de se raser les cheveux et de se rendre impur. On interprète cet ajout comme une explication théologique donnée à Jg 13-16 pour le rendre plus compréhensible ou plus « biblique ${ }^{40}$. Cependant, il faudrait pouvoir expliquer ce que «théologique» pourrait vouloir dire à propos d'un rituel de cheveux : les auteurs utilisent l'argument sans l'approfondir. En outre, le rituel tardif époque perse ( $\mathrm{VI}^{\mathrm{e}}-\mathrm{IV} \mathrm{e}^{\mathrm{e}}$ siècles) - de $\mathrm{Nb} 6$ ne correspond pas exactement à celui de $\mathrm{Jg} 13$.

Dans le texte sacerdotal de $\mathrm{Nb} 6$, les interdits sont présentés dans cet ordre :

1. le nazir doit s'abstenir de vin et de boisson alcoolisée, de même que de tout produit de la vigne ;

40. Voir Hermann-Josef Stipp, «Simson, der Nasiräer », Vetus Testamentum, $\mathrm{n}^{\circ} 45,1995$, p. 337-369, qui conteste l'hypothèse, mise en avant par les tenants de l'analyse narrative, selon laquelle Samson ne respecterait pas les interdits de nazir et donc que la mention serait indissociable du corps du récit. Pour l'auteur, Samson n'est pas un nazir - attribution tardive à partir de Nb 6 -, mais uniquement un sauveur et il suggère que le récit de sa conception aurait son origine dans le récit de la vocation d'un autre sauveur : Gédéon (Jg 6, 11-24). Les parallèles sont évidents mais ne se pourrait-il pas que ce soit en fait Gédéon qui dépende de Samson ? Voir Pierre Gibert, Vérité historique et esprit historien. L'historien biblique de Gédéon face à Hérodote, Paris, 1990, p. 54 ss. 
2. le rasoir ne passera pas sur sa tête le temps de sa consécration ;

3. il ne se rendra pas impur auprès d'un mort (vv. 3-7).

En Jg 13, 4-5, lorsque le messager divin s'adresse à la femme, l'ordre des interdits diffère :

1. tu ne boiras pas de vin ni de boisson alcoolisée ;

2. tu ne mangeras rien d'impur (v. 4);

3. le rasoir ne montera pas sur sa tête (v. 5).

Dans $\mathrm{Nb}$ 6, la mention des cheveux est insérée entre les deux interdits comme si elle était un interdit de même nature, alors qu'elle est la marque principale du nazir. Au contraire, dans Jg 13, cette mention se distingue des deux autres interdits; elle est mise en exergue. En effet, après avoir édicté l'interdit du vin et d'ingérer quoi que ce soit d'impur, le messager ajoute : « car te voici enceinte et tu enfanteras un fils, et le rasoir ne montera pas sur sa tête car le garçon sera nazir d'Elohim dès le ventre » (v. 5). Ainsi, dans ce texte, les deux interdits ne sont que la conséquence du nazir et la chevelure longue en est le signe. C'est pourquoi lorsque les interdits sont répétés par la femme à son mari Manoah (v. 7), ce troisième « interdit» sur les cheveux n'est pas répété, elle dit seulement: «car le garçon sera nazir d'Elohim». Être nazir impliquait la chevelure longue, il n'était pas besoin de le préciser une nouvelle fois. En outre, l'impureté interdite n'a pas la même origine dans les deux textes. Dans le premier, il s'agit de l'impureté due aux cadavres, dans le second il s'agit plus largement des nourritures impures. Ensuite, le vocabulaire utilisé n'est pas le même. En $\mathrm{Nb} 6$, le terme pour « rasoir » est ta 'ar, alors qu'en Jg 13, il s'agit de môrâh. De même, dans le premier texte il est dit que le rasoir ne "passera » ('âbar) pas sur sa tête, dans le second, qu'il ne «montera» ('âlâh) pas. Enfin, et surtout, $\mathrm{Nb} 6$ légifère sur le væu de nazir. Or, dans le récit de Samson, il n'est aucunement question d'un vœu ! Ainsi, s'il y a un texte qui dépend de l'autre, ce n'est peut-être pas celui que l'on croit. Ils sont pour le moins assez indépendants et reflètent, quoi qu'il en soit, deux conceptions du nazir différentes et probablement éloignées dans le temps. 
D'autres arguments sont invoqués pour montrer l'incohérence du texte présentant Samson comme un nazir et pour appuyer l'idée que le chapitre 13 est une solution théologique au récit ${ }^{41}$. Tout d'abord, on avance souvent le fait que les interdits ne concernent pas Samson mais sa mère. Il est vrai que le texte massorétique (hébreu) indique à chaque fois qu'il s'agit d'elle. Même en 13, 14, quand Manoah demande au messager la règle qu'il faudra suivre pour le garçon, le texte hébraïque comporte paradoxalement la mention de la troisième personne du féminin : «elle ne mangera pas... elle ne boira pas »; alors que la Septante a «lui ${ }^{42}$. Lorsque l'on rencontre de telles variantes textuelles, l'hypothèse habituellement retenue est celle d'une correction des traducteurs en grec afin d'harmoniser au mieux le texte. Mais l'hébreu n'est pas exempt de corruptions dues à des fautes de copistes. C'est peutêtre le cas ici car la question de Manoah est explicite et il serait étrange que le messager n'y réponde pas. Surtout, ces interdits ne s'adressent jamais véritablement à la mère. En effet, elle doit les respecter car, comme il est dit aux versets 5 et 7, le garçon sera nazir « dès le ventre». Les interdits s'adressent donc bien à Samson et, par voie de conséquence, à sa mère uniquement le temps de la grossesse. Ce n'est jamais la mère qui est concernée mais le garçon dès sa conception - quelle que soit donc la solution apportée au problème textuel posé, elle ne peut entraver le sens évident du récit.

Ensuite, il est souvent fait remarquer que Samson ne peut être nazir puisqu'il se rend constamment impur avec tous les hommes qu'il tue sur son passage. Or, il ne faut pas confondre le fait de tuer un homme sur un champ de bataille et la mort dont on méconnaît la cause pour l'attribuer à des fautes involontaires comme le contact

41. Pour cette argumentation, voir notamment Rüdiger Bartelmus, Heroentum in Israel und seiner Umwelt. Eine traditionsgeschichtliche Untersuchung zu Gen. 6, 1-4 und verwandten Texten im Alten Testament und der altorientalischen Literatur, Zürich, 1979, p. 79-111.

42. L'Alexandrinus (A) et le Vaticanus (B) ont autô(i), alors que le texte antiochien $(L)$ a autê( $i$ ). Voir Paul Harlé, Les Juges, (La Bible d'Alexandrie VII) Paris, 1999, p. 200-201. 
avec un cadavre ${ }^{43}$. Samson se rend probablement impur en mangeant un miel qui provient d'un cadavre ou en se servant d'une mâchoire encore en putréfaction, mais vraisemblablement pas en tuant des hommes au combat ou des animaux. Ce qu'il y a derrière l'impureté, ce n'est pas seulement la mort mais la contagion inexpliquée. Quoi qu'il en soit, l'interdit tel qu'il est formulé en Jg 13 n'implique pas les cas d'impureté liés directement à la mort. Enfin, on pense que la force dans les cheveux est un motif de conte étranger à la culture israélite et que le vœu de nazir permettait d'intégrer ce motif dans le champ biblique. C'est oublier que s'il n'y a pas d'autres héros semblables à Samson dans la Bible, il y a en revanche d'autres mentions de cheveux longs dans un contexte guerrier, soit concernant les tribus d'Israël (Jg 5,2), soit concernant leurs voisins immédiats (Dt 32,42). Il n'était donc pas besoin du vœu de nazir pour comprendre la figure d'un guerrier aux cheveux longs! Le vœu de nazir de $\mathrm{Nb} 6$, s'adressant autant aux hommes qu'aux femmes, est bien étranger à l'histoire de Samson. Celle-ci évoque en fait un nazir plus ancien et différent. Quelle pouvait être alors cette « institution »?

Nous avons vu que Samson est un personnage jeune, qualifié de bâchûr ainsi que ses compagnons en Jg 14, 10. Or, traduire ce terme par « jeune » ne rend qu'imparfaitement le sens hébreu. En effet, le verbe bâchar signifie «choisir, élire» et nous trouvons souvent l'expression 'îsh bâchûr dans des contextes guerriers pour désigner un corps d'élite. Par exemple, quand Saül traque David, il réunit trois mille hommes, l'élite d'Israël (1 S 26,2) - Saül luimême est qualifié de bâchûr (1 S 9,2). De même, David rassemble «toute l'élite d'Israël» afin de récupérer le coffre de Yhwh (2 S 6,1). Mais la mention de bachûrîm la plus intéressante se trouve dans le récit de la guerre opposant la tribu de Benjamin aux autres tribus d'Israël. Il y a des deux côtés, dans chaque armée, des « hommes d'élite», sept cents sur vingt-six mille hommes en tout

43. Le texte de $\mathrm{Nb} 6$ prévoyait une procédure de purification (vv. 9-12), qui intégrait le rituel de la vache rousse permettant d'obtenir une eau lustrale avec les cendres de celle-ci ( $\mathrm{Nb} 19)$, tant l'impureté était omniprésente dans l'esprit sacerdotal ; rien de tel en Jg 13-16. 
du côté de Benjamin $(\operatorname{Jg} 20,16)$ et dix mille pour quatre cent mille hommes en tout du côté des autres tribus (Jg 20, 17 et 34). Si nous ne pouvons pas tenir compte des chiffres en terme de nombre, en revanche, nous pouvons retenir que la part des soldats d'élite pour l'ensemble des deux armées est faible : plus que des soldats, ce sont des guerriers. Mais le plus intéressant se trouve dans leur description. Les Benjaminites sont dits «liés de la main droite, tous ceux-là lançant la pierre pour un cheveu et sans manquer » $(\mathrm{Jg} 20,16)$. Ils ne sont donc pas des soldats armés lourdement, ils se battent avec une fronde et sont extrêmement habiles. Cela n'est pas sans rappeler le jeune David qui préfère sa fronde et son bâton à l'armure et à l'épée de Saül (1 S 17,38-40). Le modèle du jeune guerrier est dans ces descriptions. Quant au bras droit lié, il peut être un jeu de mot sur le nom de la tribu de Ben-yamîn, « fils de la droite », mais tout autant une pratique effective de cette tribu. En effet, Ehoud le Benjaminite est dit aussi « lié de la main droite » et c'est par ruse qu'il tue le roi de Moab, saisissant son poignard de la main gauche sur sa hanche droite (Jg 3, 15 ss). Le jeune guerrier, le bâchûr, était donc l'opposé du guerrier confirmé, par la légèreté de son armement et par sa manière déloyale de combattre.

Le lien entre la figure de Samson et la figure du jeune guerrier existe bien, même si le héros a une ampleur tout autre encore. Mais comment établir le lien entre le jeune guerrier et le nazir? Les mentions de nazir dans la Bible hébraïque sont si rares qu'il est bien délicat de le vérifier. Toutefois, dans le livre d'Amos, il y a une mention importante : «j'avais fait lever parmi vos fils des prophètes et parmi vos jeunes (bachûrîm) des nazirs » (Am 2,11). Même si ce verset fait partie d'un ensemble considéré comme deutéronomiste ${ }^{44}$, il reste que cette évocation précède le texte sacerdotal de $\mathrm{Nb} 6$ et renvoie à une époque ancienne - l'époque monarchique et pourquoi pas pré-monarchique! Or, il n'est pas question d'un vœu de nazir concernant les hommes et les femmes mais d'une « institution » ne

44. Ceci depuis l'article de Werner H. Schmidt, « Die deuteronomistische Redaktion des Amosbuches. $\mathrm{Zu}$ den theologischen Unterschieden zwischen dem Prophetenwort un dem Sammler », Zeitschrift für die Alttestamentliche Wissenschaft, $\mathrm{n}^{\circ} 77,1965$, p. 168-193. 
s'adressant qu'aux jeunes hommes; ce qui n'est pas sans rapport avec Samson. Certes, la référence à la guerre n'est pas du tout évidente, même si le rôle des prophètes n'y était sans doute pas étranger à l'origine : il suffit de penser à Debora ${ }^{45}$. Cependant, il s'agit bien d'une évocation positive des « anciens » prophètes attachés aux temples - que l'on trouve notamment mentionnés dans les textes de Mari (première moitié du $\mathrm{II}^{\mathrm{e}}$ millénaire) -, à distinguer des «prophètes écrivains » qui se montrent hostiles le plus souvent à leur encontre ${ }^{46}$. L'ancienneté et le lustre de la classe des prophètes étant sous-entendue dans ce passage, l'institution de nazir ne peut être nouvelle non plus.

Il reste encore deux aspects à évoquer, l'un concernant l'étymologie du mot, l'autre étant un éclairage anthropologique. Contrairement à ce que l'on a parfois avancé, se fondant sur l'emploi de $n z r$ dans une inscription araméenne ${ }^{47}$, le verbe nâzar n'est pas l'équivalent de nâdar, "vouer». On comprendrait d'ailleurs mal pourquoi en $\mathrm{Nb} 6,2$, pour le vœu de nazir, on ait les deux verbes associés s'ils étaient strictement synonymes. On peut penser en effet que le terme de nazir ait pris progressivement un sens plus général de «consacré », mais le sens originel de la racine est " couper, séparer » ${ }^{48}$. Or, de même que le crypte lacédémonien se comprend par la traduction de kruptos, « caché », le nazir pourrait mieux être saisi si nous traduisions par le «séparé » - et Samson est bien le personnage qui est séparé, coupé de la société, errant et

45. Weber a développé toute une interprétation du lien unissant prophètes et nezirim dans la guerre, dressant à grands traits l'évolution sociologique supposée d'Israël. Max Weber, Le judaïsme antique, Paris, 1970 (Tübingen, 1920), p. 134-168.

46. Voir Francolino J. Gonçalves, «Les "prophètes écrivains" étaient-ils des nby'ym ? d dans M. Daviau et alii (eds.), The World of the Arameans I, Sheffield, 2001, p. 144-185.

47. C'est ce qu'atteste la stèle du IX ${ }^{\mathrm{e}}$ siècle de Bar-Hadad dédiée au dieu Melqart. Voir William F. Albright, « A Votive Stele erected by Ben-Hadad I of Damascus to the God Melcarth », Bulletin of the American School of Oriental Research, $\mathrm{n}^{\circ} 87,1942$, p. 23-29.

48. Pour une analyse détaillée, voir Jesse L. Boyd, «The Etymological Relationship between NDR and NZR reconsidered », Ugarit-Forschungen, $\mathrm{n}^{\circ} 17,1986$, p. 61-75. 
vivant aux marges. En outre, si l'on considère que le $n$ est incrémenté à $z r$, prenant cette base comme un équivalent à la racine en indo-européen ${ }^{49}$, nous retrouvons un sens général de séparation avec des radicaux comme $z w / y r$ ou dans des termes formés à partir de $z r$, qu'il s'agisse de sens «doux » comme «s'éloigner» ou de significations plus brutales comme «presser, fouler », voire « haï, ennemi... ${ }^{50}$. Ainsi, il y a peu de chances pour que le nazir ait été le consacré au sens de celui qui était voué - séparation « positive »-, mais bien plutôt celui qui était tenu à l'écart des autres membres de la société, au moins pour un temps. Et c'est là que nous retrouvons la possible marginalisation des rites de passage.

Enfin, l'argument anthropologique qui permet de lier le nazir à la figure du guerrier avec une grande probabilité, c'est le motif de la chevelure longue. Sur les champs de bataille, les ennemis apparaissent avec des « cheveux longs » (Dt 32, 42), de même les tribus israélites laissent leurs « cheveux libres » pour combattre $(\operatorname{Jg} 5,2)^{51}$. Samson, modèle de guerrier, se définit aussi par une longue chevelure : le symbole de sa force sauvage est entièrement contenu dans cette chevelure $^{52}$. Et il est nazir, c'est-à-dire qu'il lui est interdit de se couper les cheveux. Il y a forcément un rapport entre le héros aux

49. Conformément à une nouvelle théorie sur les langues sémitiques mettant en évidence, notamment, un étymon bilitère originel à la place de la traditionnelle racine trilitère. Voir pour cette théorie Georges Bohas, Matrices, étymons, racines. Éléments d'une théorie lexicologique du vocabulaire arabe, Leuven-Paris, 1997 et Matrices et étymons. Développements de la théorie, Lausanne, 2000.

50. Voir David Cohen, Dictionnaire des racines sémitiques, II, 8, Leuven, 1999, p. 709-711 et 788 ss.

51. Dans l'Iliade d'Homère, les guerriers Achéens sont souvent qualifiés de « chevelus ». La chevelure peut d'ailleurs être arrangée de différentes manières. Les Abantes dans l'épopée homérique, dont il est dit qu'ils respiraient la «fureur » (ménos), semblent être rasés sur le devant de la tête mais avoir les cheveux longs sur la nuque (Iliade, II, 536 ss.).

52. Gilgamesh porte également la chevelure longue : "les touffes de sa chevelure étaient drues [comme celles] de la déesse des orges » (T. I, $\S 4$, II, 6-7) ; «Gilgamesh lava sa tignasse, nettoya son bandeau, secoua sa chevelure sur son dos » (T. VI, § 1, I, 1-2). Cuchulainn a, quant à lui, une chevelure longue, tressée et de trois couleurs différentes : littéralement, il a trois chevelures ! Voir Bernard Sergent, Celtes et Grecs I. Le livre des héros, Paris, 2004, p. 138. 
longs cheveux et les guerriers aux chevelures spécifiques, et il est probable que ce soit le nazir. Le rapport doit même être direct - nazir-cheveux longs-force - et non une simple illustration à partir d'un rite cultuel qu'est le vœu de cheveux. Il est à noter que le texte de $\mathrm{Nb} 6$ garde d'ailleurs une trace de l'ancienne conception puisque celui qui se vouait nazir devait avoir la chevelure libre (v. 5), à l'instar du lépreux $(\operatorname{Lv} 13,45)$ et à la différence des prêtres (Lv 21, 5 et 10) - même si l'auteur sacerdotal, en $\mathrm{Nb} 6,5$ et 8, lui a attribué la qualité d'être « sacré » (qâdosh), les cheveux sauvages le situaient donc encore plus proche de l'aspect de l'impur que de celui du consacré.

Ainsi, tous les indices relevés montrent la cohérence qui existe entre le héros Samson et une «institution» de nazir difficile à cerner mais probablement liée à la guerre et aux jeunes guerriers, par notamment la chevelure sauvage ${ }^{53}$. Il faut toutefois convenir que les attestations de tels nazirs liés à la guerre sont quasiment absentes des textes. Nous avons vu que Samson, par certains aspects, se rapproche des grands héros des épopées et incarne, par d'autres aspects, la figure du grand guerrier, semblable aux gibbôrîm (preux, héros) de David (2 S 23, 8 ss.). Mais celle du jeune guerrier échappe quelque peu à nos investigations et si les bachûrîm benjaminites ou si le jeune David paraissent s'en rapprocher, rien n'indique une relation directe avec le nazir. Nous n'avons, pour établir cette relation, que le bref passage du livre d'Amos. Ce texte suggère d'ailleurs une solution : relier le nazir à la manifestation de l'esprit de Yhwh. Il est vrai que le récit de Jg 13-16 semble refléter deux rédacteurs différents, l'un parlant de Samson nazir (Jg 13 et 16), l'autre expliquant sa force spectaculaire par l'intervention de l'esprit de Yhwh (Jg 14-15). D'un point de vue textuel, tout rapprochement entre les deux ensembles est donc incertain. Cependant, la mention d'Amos qui énonce que Yhwh faisait lever des prophètes et des nazirs permettrait d'étayer l'hypothèse selon laquelle l'esprit de Yhwh s'emparant de Samson s'expliquerait par sa qualité de nazir. En effet, si on ne peut savoir comment le dieu faisait lever des nazirs,

53. C'était déjà la conviction de M. Weber, Judaïsme antique, p. 139-140. 
on sait en revanche comment il procédait pour les prophètes : par son esprit !

Quoi qu'il en soit, nous pouvons avancer l'idée qu'il existait dans l'ancien Israël (pré-étatique) une élite de guerriers. On ne peut se contenter de penser que les tribus « israélites » ne se battaient qu'avec leur seule témérité 54 . Même si les textes en disent peu, il est fort probable qu'existait une «institution » de jeunes guerriers et que le mot nâzîr la désignait ou, plus sûrement, pouvait désigner certains d'entre eux. Il reste que l'histoire de Samson nazir se comprend mieux ainsi plutôt que comme référence à un vœu temporaire - le rédacteur pré-deutéronomiste de Jg 13-16 ne pouvait ignorer l'écart de sens entre un héros guerrier comme Samson et un simple vœu sur les cheveux ${ }^{55}$. La seule inconnue restant la nature précise du nazir: simple rite de passage des jeunes hommes ou « consécration » guerrière de quelques-uns ? Samson semble nous indiquer qu'il s'agit de la seconde possibilité.

Au terme du parcours, nous pouvons conclure :

1. L'histoire de Samson peut difficilement être attribuée à la culture « grecque » - ce qui n'interdit pas la présence d'éléments de cette culture - tant les parallèles avec d'autres héros non-grecs, et notamment Gilgamesh, sont évidents. Non seulement Samson est typique de la figure du héros, grec ou autre, mais en outre il a les traits de grands guerriers contenus dans la Bible hébraïque.

2. L'unité de l'ensemble du récit est apparemment évidente, mais diverses mains ont pu intervenir dans l'actualisation du texte.

54. « Il y a des coutumes de guerre et des règles de combat, mais il n'y a pas une organisation militaire stable. »; «Ces troupes réduites mais bien tenues en main par leurs chefs venaient à bout d'ennemis plus nombreux ou mieux équipés par des attaques hardies, des ruses de guerre, des embuscades. ", R. de Vaux, Les Institutions de l'Ancien Testament II. Institutions militaires, institutions religieuses, Paris, 1991 (1960), p. 11 et 15.

55. Josèphe, percevant cet «écart », s'est gardé de faire mention du nazir et a assimilé Samson à un prophète, jugeant l'écart moins important (?) ; surtout parce que, juste avant, il avait défini le vœu de nazir d'après $\mathrm{Nb} 6$. Voir Flavius Josèphe, Antiquités Juives, IV, 72 et V, 275-317. 
À l'arrière-plan, on discerne encore les exploits d'un surhomme aux allures de géant, héros lié au dieu Shamash. L'adaptation yahwiste qui en a été faite - en plusieurs temps probablement - a permis d'introduire le nazir. Pour finir, il a été intégré tardivement dans le livre des Juges et n'a «subi » qu'un simple encadrement deutéronomiste, sauvegardant sa nature de récit héroïque.

3. Par le lien qui a été fait entre le héros et un rituel de nazir, nous disposons d'une source essentielle au sujet d'une «institution » de jeunes guerriers, toutefois très difficile à cerner; il reste que le lien entre Samson et le vœu de nazir est trop distendu pour penser qu'un rédacteur final ait inséré cet élément afin de donner un sens à cette histoire de cheveux : Jg 13-16 et Nb 6 parlent de deux nazirs distincts.

4. Ce récit peut être appelé un mythe, non au sens des grands mythes cosmogoniques mais au sens d'un récit d'origine ancienne, proche du conte et comportant des éléments religieux. Au sens aussi d'un récit non encore inscrit dans le temps de l'histoire malgré sa place dans un livre de nature historique ${ }^{56}$. L'introduction d'éléments rituels dans le récit nous amène même à conclure qu'il s'agit d'un mythe étiologique ${ }^{57}$.

34, rue de la Montagne Sainte Geneviève

75005 Paris

ch.lemardele@laposte.net

56. Pour une telle définition du mythe, qui s'oppose à une conception historique du temps, voir Claude Lévi-Strauss, « Le temps du mythe », Annales $E S C, \mathrm{n}^{\circ} 26,1971$, p. 533-540.

57. Je remercie Étienne Nodet, Jean-Baptiste Humbert et Paolo Garuti qui ont accepté de faire une lecture critique d'une première version de ce travail lors de mon séjour à l'École biblique et archéologique française de Jérusalem en 2002-2003. Je remercie aussi, vivement, Pierre Vidal-Naquet: il a eu la gentillesse de lire ce texte (qui doit beaucoup au Chasseur noir) et m'a fait des remarques enthousiastes. 